\title{
The Future of Software Engineering by 2050
}

\author{
Dr. Saada Abdelgawad Mohamed Khadragy \\ The British University in Dubai \\ 20170009@student.buid.ac.ae
}

\begin{abstract}
According to the huge criticality of using advanced software systems and the great demands of providing updated and qualified programs, this paper reflects some future perspectives towards the importance of software in managing everything in the world life over the next thirty years. Moreover, the paper presents the challenges which face the recent and the future generations as a result of this huge revolution of technology and automations. In addition to that, the writer would provide some solutions for those obstacles in order to enhance the role of software engineers in 2050. Also the paper reviews the related literature and surveys the point of view of software engineering experts by assigning an online interview with six open ended questions to examine their perspectives towards the future of Internet of things, Artificial intelligence, ubiquitous computing, and system of systems. The majority of ideas reflected the humanity fears of technology and replacing people with robots in the job fields. Furthermore, some experts encouraged the idea of having smart homes, and wearable devices
\end{abstract}

Keywords: advanced Software; system of systems; 3D building printer, internet of things, artificial intelligence, ubiquitous computing, and system of systems.

DOI: $10.7176 /$ CEIS/11-2-06

Publication date: April $30^{\text {th }} 2020$

\section{Background}

Between the recent days and 2050, there will be many changes and the capacity of institutions and their services, programs, and systems will follow an advanced generation of software. On the other hand, there will be significant and unexpected issues to the worldwide range even economically or politically related to the revolution of industry and the rapid increase in number of people and their needs.

This paper particularizes the nature of these cumulative demands of software systems and their associations. The paper introduces both of importance and challenges of the future of software engineering through four sections; section one presents a general view about the use of IOT(Internet Of Things) in the recent and the future days, section two introduces the term of Artificial Intelligence, its importance and future, section three would be about Ubiquitous computing and its importance, and section four would be about system of systems. All of the four areas would be examined from the point of view of some experts and practioners. Finally, the researcher would conclude the paper with general point of view and suggestions.

Accordingly, software has become a progressively important and energetic element of our society. There is no commerce field or factor of our daily life which is not controlled by software. Individual and group tasks, business and economic enterprises, political and manufacturing substructures, governmental, educational, and amusement are all intensely infused and ruled by software applications and systems. Thus, software growth has become an essential task that should be sensibly considered, understood, deliberated, and improved [1].

In the past fifty years, the main purpose of the Software Engineering community has been to improve and manage these matters. Also, the software engineering group emphasized the way of illustrating, identifying, and enhancing the methods, rules, and systems to control this complicated issue.

Also, the research in the field of software engineering provided many remarkable results; some of them have been exaggerated, while some others were ignored. So, no one can deny that research in the field of software engineering has many issues and procedures are still under investigation [2].

However, there are many papers written in solving the software products issues, one paper presented a survey about some attempts to collect many articles related to software products line. This research focused on ideas of improving the present advances in genetic improvement which helps in growing new products with new functional and non-functional techniques [3].

The recent study is divided into four areas. The researcher tended to survey them based on reviewing the literature. The main four areas of this paper are internet of things, artificial intelligence, ubiquitous computing, and system of systems. The following section will be about those four areas, their present and future. 


\section{Literature Review}

In this section, we would review some historical resources, articles, and perspectives about the future of software engineering and its future obstacles. In one study, the writer described the developed global future with explaining the challenges that face achieving a certain vision and he reviewed some related topics from the literature, reflecting challenges in four areas; software engineering, ways of communication and their requirements, environment and equipment. Then, the study was concluded by recommending having a systematic understanding of our needs and what drives the society [4]

On the other hand, Software systems shape the basics of the new information community, and many of those systems are between the most complicated items ever produced. Software engineering (SE) is about improving, preserving and controlling high-quality software systems in a profitable and expectable method. SE research examines the real-world phenomena of SE and worries [5] the enhancement of modern, or adjustment of existing, technologies to help SE tasks, and [6] the assessment and contrast of the effect of using such technology in the often very complicated interaction of persons, groups, projects and institutions, and different kinds of activity and software system.

Another study cited the short history of software engineering. Software growth has been categorized by dangerous separates between important tasks such as planning, organizing and application. The problem is further intensified by the sporadic and rare achievement of activities such as planning, examining, mixing and announcement. Several mixing phenomena introduce efforts to emphasize these issues. For instance, endless incorporation is an activity which has arose to remove breaks between progress and distribution. We suggest a similar steadiness is obligatory between business approach and progress [7]. These separates are even trickier assumed the need for dependability and flexibility in the complicated and data-intensive systems being improved in the recent days. We determine a number of nonstop activities which together we call as Continuous which we introduce as part of an overall view for Continuous Software engineering [8]. To reach this goal, scientists need to understand and identify the demands of people and societies. For instance, one researcher developed a model to examine developers' status of mind [9].

\section{The Internet Of Things:}

The Internet of Things (IoT) signifies a broad setting that includes a huge number of devices intersecting mixed physical items to the Internet. As it is clear in Fig1, physical items are connected together to construct the IOT platform. Many areas such as industrial, farming, cultivation, automated homes, and many ubiquitous computing applications have used IOT [10]. As Fig 1 shows many different fields need that connection between their devices to qualify the presented services.

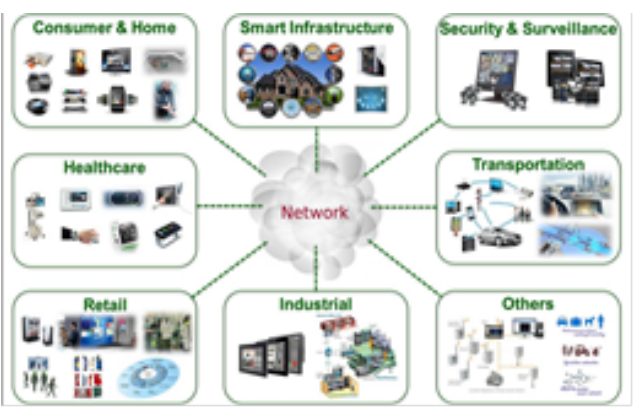

Fig1. The IOT platform.

Also Internet of Things (IoT) is a talented technology that aims to transform and link the universal world through assorted smart devices through unified connectivity. The recent need for machine-type communications (MTC) has risen in a diversity of communication technologies with different service tools to attain the modern IoT foresight. More recent cellular principles like long-term evolution (LTE) have been presented for mobile equipment but are not well instructed for low-power and low data rate equipment such as the IoT devices. To tackle this issue, there is a number of developing IoT standards. Such as, Fifth generation (5G) mobile network 
which aims to adopt the restrictions of old cellular features and be a probable key enabler for future IoT. In one paper, the state-of-the-art of the IoT application needs along with their related statement technologies is reviewed [11].

The following line graph reflects the high increase of using IOT and the number of research papers was written in 2016/2017 to investigate the benefits of IOT in different fields. The presented indications and evidences in the recent years are considered as an evidence for the future use depending on the nature laws for following the same directions by passing the time.

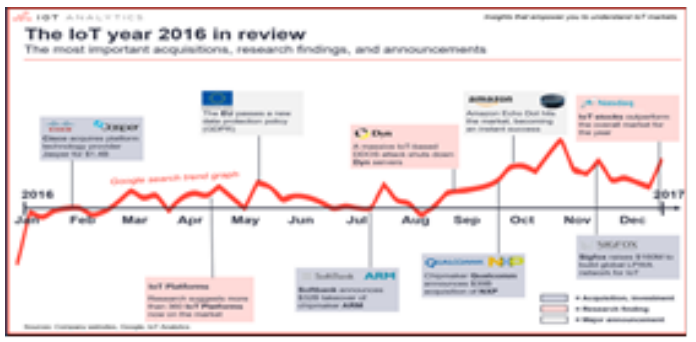

Fig2. Reviewing the improvement of IOT in 2016.

For example, the overview of mobile devices has revolutionized our day-to-day lifetimes. They allow consumers to attain information even in an itinerant setting and give information without constraints. After 100 years the overview of this technology, but we are now confronting the next revolution that will transform our day-to-day lifetimes. With the insertion of the Internet of Things (IoT), our interaction would not be limited to only mobile devices. Sooner, it will enlarge to include all services with which we live. A lot of research papers have examined IoT-related stages and platforms. In addition, there are only few arguments about the IoT network [12].

As the following figure explains the main parts and tasks of IOT. Any system is a mixture of software and hardware to achieve an enthusiastic task. Some of the major devices used in fixed services are Microprocessors and Microcontrollers. Microprocessors are normally known as general objective processors as they usually receive the orders, process it and give the results. While a microcontroller not only receives the data as inputs but also operates it, shares the data with different devices, manages the data and then finally gives the outputs.

\section{IoT Platforms}

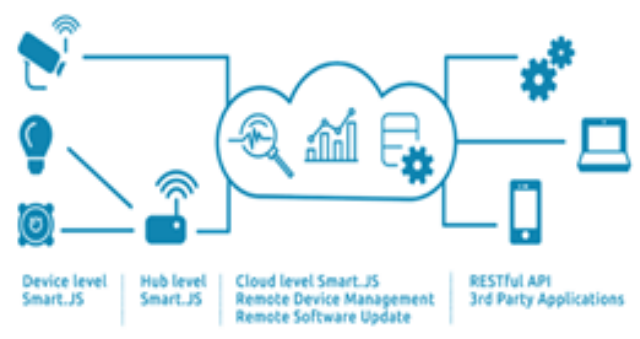

Fig3. IOT platform and work.

On the other hand, Internet of Things (IOT) introduces an essential role in connecting the surrounding environmental objects to the network and get easy to access those concrete objects from any remote location. It's unavoidable for individuals to know the increasing technology. And usually people have issues on parking vehicles in parking times in a city. In one study the authors plan a Smart Parking System (SPS) which allows the user to reach the nearest parking area and provides availability of parking times in that particular parking area. And it mostly focus on decreasing the time in reaching the parking lots and also it keeps away from the 
redundant travelling through occupied parking lots in a parking place. Also it decreases the fuel consumption which also decreases carbon footprints in an atmosphere [20].

\section{- $\quad$ Artificial intelligence:}

$\mathrm{AI}$ is the second area of this research paper. As people live in a time of inconsistency. Organizations applying artificial intelligence complement or exceed people point achievement in many areas, achieving fast enhancements in other fields and driving high stock values. Since calculated productivity development has decreased by half of the past centuries, and usual income has deteriorated meanwhile the late 1990s for a maturity of Americans. We label four possible clarifications for this conflict of prospects and numbers: wrong expectations, false measurement, restructuring, and employment gaps [13].

The numerical revolution is in its greatest generation. The question is about the way of the change which will result from this revolution. The quantity of data we generate twice as much every year. It means that in 2016 we generated the greatest amount of data in the whole history of our lives through 2015 . Every minute we generate millions of Google searches and Facebook posts. This includes material that conveys the way of our thoughts and emotions are built. In the future, everything in our lives, conceivably even our clothing, also may be linked to the Internet. It is approximated during the 10 years' time there will be 150 billion interacted computing sensors, more than 20 times population on our planet. As a result, the quantities of data will amplify every 12 hours. The majority of institutions are already trying to change this big amount of Data into Big amount of Money as a kind of industry. We think that all things around us will be intelligent; also we will not only have smart devices, but also smart houses, smart institutions and smart places. As a result, we expect smart nations to be one of the main goals of the field of artificial intelligence which is, actually, producing spectacular developments. Especially, it is donating to the automation of data analysis. Artificial intelligence is not constructed step by any one, but is now able to learn, thus uninterruptedly improving itself. Newly, Google's Deep Mind algorithm learned itself how to gain 49 Atari games. Algorithms can now realize handwritten concepts and designs almost as well as people and also conclude some activities better than humans. They are capable of labeling the subjects of images and videos. In the recent days, $70 \%$ of all commercial industries are achieved by algorithms. For instance, the major part of the news is automatically produced. All of this has fundamental financial significances: in the coming 10 to 20 years about half of today's jobs will be reduced by algorithms. $40 \%$ of today's major 500 institutions will have endangered in a decade. It can be supposed that new generations of computers will soon exceed human abilities in the majority of fields. - wherever between 2020 and 2060. Specialists are beginning to call alarm signs. Technology prophets, such as Elon Musk from Tesla Motors, Bill Gates from Microsoft and Apple co-founder Steve Wozniak, are alerting that super-intelligence is a critical risk for humanity, probably even more serious than nuclear weapons. One thing is significant: the approach in which we systematize the economy and society will vary basically. We are feeling the major alteration since the end of the Second World War; after the mechanization of manufacture and the formation of self-driving cars the computerization of society is coming soon. As a result, humanity is at great point, which assures great chances, but also significant dangers. Then, when we decide to take any decision, we have to be aware of its results because it will affect our future and threatened our past performance. No one can deny the fact of past inventions development in both of the present and future. In the last decade, Norbert Wiener (18941964 ) originated cybernetics. He insisted that behaviour of systems could be regulated by the tools of appropriate feedbacks. Immediately, some researchers assumed managing both of the economy and society based on this simple rule, but the essential technology was not accessible at that time.

\section{- Ubiquitous computing:}

The huge spread of processing into the natural fields assures more than the ubiquitous obtainability of computing substructure; it provides new examples of communication enthused by continuous approach to instruction and computational competences. Through the past few decades, many research papers indicated the importance of applying ubiquitous computing (ubicomp) which has argued three communication areas: usual boundaries, framework based, and robotic apprehension. To monitor the progression for future research in ubiquitous computing, we revise the activities of these labors and direct the other research obstacles. Research in ubiquitous computing indirectly needs to find out some concepts of rule, not only in the amount of devices, the substantial space of spread computing, or the number of population using a system [14].

There are many fields those will be affected by the technology of ubiquitous computing, such as business and industries. Ubiquitous computing will shift the style we survive with technology. Mark Weiser illustrated that: 
"The most insightful technologies are those that vanish. They include their roles into the stuff of the daily lives until they are identical from it" [15].

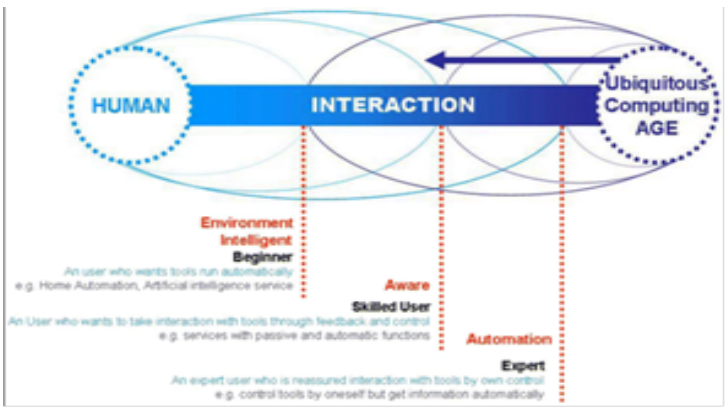

Fig4. Interaction between human and UBICOM.

Figure 4 indicates the interaction between people and machines and introduces the three components of this interaction (environmental intelligent, aware, and automation). Also, we don't consider that pens or joints or spouts as technology. They are just basically structures of the world we live in and construct the technique we behave in the life. With ubiquitous computing, enhancing information technology will gradually bear more like applying these everyday elements than applying personal devices [16]. The tendency to ubiquitous computing does not introduce just a modification in the way of contacting and applying information. Eventually, that would have a deep impact on the way people contact and apply services, allowing new generations of services that only have sense by feature of being rooted in the environment [16].

\section{- $\quad$ System of systems:}

The aim of the Tip Framework is to capably push the performance, achievement and process of unified, joint systems "as it is shown in pic.3". It is depends on the presented Service. The basic components of the framework are systems that introduce and utilize services, and work as systems of systems. Some usually implemented systems, such as instrumentation, approval or service archive are called as essential.

\section{System of Systems}

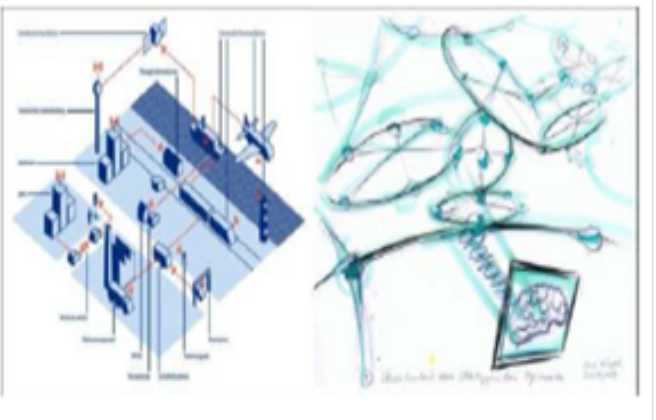

Fig4. System of systems platform.

These can be applied by any system of systems that have the instructions of the Arrowhead Framework. Within the framework, systems - applying varied information change technologies throughout collaboration - are contributed through many approaches. In addition to what is called Interoperability stage, as well as systems and services for transformation. Moreover, one of the basic issues of improving such extremely interoperable 
systems is the shortage of sympathy between different development communities. Suitable improvement and service certification ways can contribute to overwhelm this issue. The structure, improvement and confirmation methodology for each task, system and system of systems within the Arrowhead Framework helps that these can be applied, confirmed, organized, and run in an interoperable way [17]. The term of system of systems is considered as a practical solution for many technological issues. As International Institute for Applied Systems Analysis (IIASA) presented policy-oriented research, according to integrated systems explanation of normal, technology and substructure and population and social systems to obtain clarifications for sustainability alterations. The Governance in Transition study theme within the Risk and Resilience program explores how governance structures form ideas and successive consequences by constructing and contributing to research on decision-making strategies, general agreement, risk insight, cognitive preferences, and national perspectives, as well as participating governance project [18]

Methodology:

The recent paper introduces a clear view about the future of software engineering by 2050 through main four areas. Also it presents our conceptual framework of the future challenges which will be resulted from the development of IOT, AI, UC, and SOSs.

An online interview was constructed for a number of expertise and the questions of this interview were assigned to some CS experts from different places in the world through Skype and linked-in applications.

Six questions were formed as followed:

Q1. What would be the future of software engineering by 2050 from your own point of view?

Q2. How would the software system affect the future of Artificial Intelligence and Big Data?

Q3. How would the software system affect the future of 3D Printing and consumer products as an example of Artificial Intelligence?

Q4. Explain your own point of view towards the impact of advanced software in the future of Driverless cars.

Q5. What would be the relationship between the services and the costs of software systems in the future?

Q6. In your opinion, what would be some of the negative impacts of the Internet of Things (IOT)?

Through the next section, we would analyze the answers of the questions from the experts and scientists in the field of software engineering and computer science.

Data analyses:

The previous section presented the online interview questions which were assigned to a number of scientists and experts.200 invitations had been sent to CS specialists to ask them if they would be able to participate in the mentioned online interview in order to survey the majority of ideas and perspectives towards the future of software engineering. 85 expert accepted to participate and give answers for the six questions. Lastly, 72 experts only sent their answers. The following explanation gives the ideas of the majority of the participants:

Q1. What would be the future of software engineering by 2050 from your own point of view?

The majority of answers came to support the idea of Software Engineering differs from all other branches of engineering because it is in constant evolution. The concepts that seem relevant today can become completely redundant in the next few years. Also they feel that the software would become a very integral part of our lives in the near future. From self-driving cars to wearable devices, the amount of technology immersed in our environment would make us a very different species as compared to our past generations. Along with the technology, the environment must evolve as well. For example if we have a self-driven car, the traffic lights installed by the government and the way fines are dealt with would have to change as well. The point we are trying to make is that the future of software engineering is not just confined to software itself, but it will force the environment around us to change at a very rapid pace.

Q2. How would the software system affect the future of Artificial Intelligence and Big Data?

We believe that the bulk of scenarios where Artificial Intelligence would be put to use are in places or jobs that are too dangerous for humans to do. Robots are already taking over some of the most hazardous jobs available, including bomb defusing. Other jobs such as welding, well known for producing toxic substances, intense heat, and noise, would likely be outsourced to robots in most cases. Machines have much more access to data than one 
person ever could - storing a large amount of statistical information. Using big data, Artificial Intelligence systems could one day identify trends and use that information to come up with solutions to the world's biggest problems, such as climate change. Additionally, 'Home' robots or domesticated robots could help elderly citizens with everyday tasks and allow them to stay independent for as long as possible, which could improve their overall well-being.

Q3. How would the software system affect the future of 3D Printing and consumer products as an example of Artificial Intelligence?

We think that what we are seeing right now is just the start of a big revolution in the world of manufacturing. The day we get to a point where we can use Metal for 3D printing, it would change of the world of consumer electronics. The prices would drop significantly because if the 3D printers are affordable, families could start printing their own tools to avoid hefty market prices. Same is the case with 3D printed parts of the human body, such as bones, or valves. That would surely change the world of healthcare and would make things much more affordable in the long run. Some experts stated that "Personally, we feel that 3D printing would have a much greater impact than any other technology mentioned in this interview". We could add that there is Some Increasingly Significant Technology by 2050 for the European Commission's KT 2050 scenarios group as the relation between different technology fields is indicated in the following table:

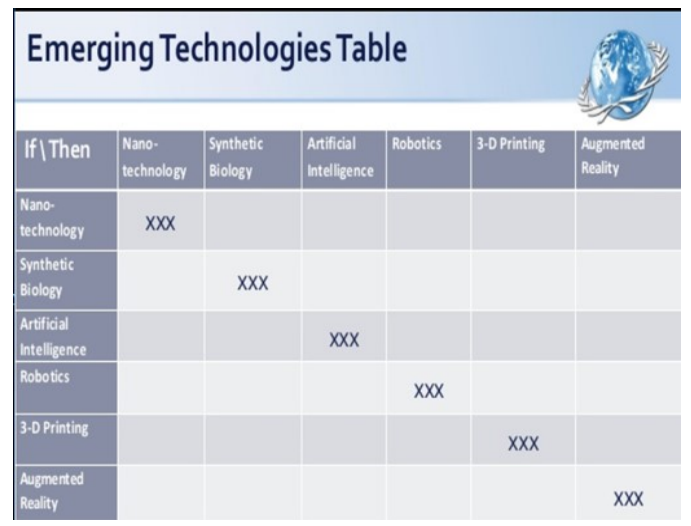

Fig5. Emerging technologies table.

Q4. Explain your own point of view towards the impact of advanced software in the future of Driverless cars.

As the population of the world is increasing at a very rapid pace, transportation would be a big problem in the future. The amount of traffic on the roads, along with new laws and fines would incline people towards Driverless cars. As vehicles become smarter and more automated, there will also be advances in data communication between cars on the road and between cars and roadside installations. In other words, there is a need to "teach" those cars to take into account real-time data about their surroundings and other vehicles. This would require massive investments from governments around the world, something that I remain very skeptical about.

Q5. What would be the relationship between the services and the costs of software systems in the future?

With the amount of technology around us, the demand for software engineers with current and relevant skills would go higher. This would result in higher salaries in the technology industry, which could lead to higher cost of software. However this can be countered by introducing emerging markets such as China, India, Pakistan and Brazil into the mix, since they cost of development is lower in these countries. Secondly, I think the concept of open-source software would gain more attention and this would enable people to have free alternatives as well.

Q6. In your opinion, what would be some of the negative impacts of the Internet of Things (IOT)?

One word: Privacy. We believe that we have already surrendered this aspect of our lives to various social media platforms and corporations such as Google and Microsoft, which control most aspects of our digital lives. However if the Internet of Things concept gets more popular in the future (and we are sure it will), then there would be an increased need of security and privacy algorithms to protector us against hacking, unauthorized access and identity fraud. As adults, by and large, we guess that our houses as very confidential and private places ... for children it's not a private place. They have no power over who enter and leave their rooms, or who enter and leave their houses. As a result, the technological online world gives more privacy because it looks like 
it has more control [19].

The results:

In addition to the previous analysis, I believe in having a smart generation of everything which will affect the future of software engineering positively and negatively. The positive side would be in providing easy, comfortable, and costless services. While the negative side would come clear in reducing the number of people in each institution and company because they would be replaced by machines and robots.

I think that the major ten cycle trends for the future of software engineering would be as: the industry will be gradually based on innovation and both workers and consumers would be less exhausted. Then, the form and offer of the infrastructure industry will move extensively, with new business forms, artifacts and products. Accordingly, Infrastructure will be reshaped on form physical and steel to contain new resources which greet their environments. Also, new careers and industries will be generated and some will vanish, particularly low or zero skill roles and those depending on repetition of activities. Caring only about design and construction will be an outdated concept as infrastructure becomes multi-functional. Moreover, robots will become more predominant in construction. As a result, construction will get sooner, using 3D and 4D printing, and selftransforming elements which self-assemble. New, troublesome thoughts will arise, for making mass transportation faster, saved and less harmful to the environment. Thus, we will gradually use more wearable technology such as exoskeletons. Lastly, direct neural regulator over devices and vehicles will be connected to the industry.

On the other hand, The Internet is successively transformed and developed. The basic connection shape of recent Internet is human-human. The Internet of Things (IoT) can be determined as the future evaluation of the Internet that recognizes machine-to-machine (M2M) learning. Consequently, the internet introduces connectivity for everybody and everything. It implants some intelligence in Internet-connected items to interact, transforms information, take decisions, appeal actions and support smart services. All of this lets software engineering services gradually beneficial and software vocations progressively powerful, but it also targets substantial accountabilities on software engineers to certify that their software will be able to provide great amount of dependability. Also there are some extra future goals that would make this goal progressively challenging, but also gradually essential to tackle. These goals are: ambiguity and development; quick transformation; complex dependability; variety; and interdependence.

The improvement of computing technology touches the environmental transformation of domestic field a lot in a many technologies. It means that we suggest that Ubiquitous computing technology will shape our very concept of our houses. The most dissimilar occurrence of Ubiquitous settings is that the amount of individual computers will enlarge main fold. So in the coming future we will collaborate with computers in daily life and people will have more computers to control. Related to users, the interaction between systems and users in home-network setting should construct the next stage which is distinctive from recent way of using computer. Hence, Ubiquitous setting should be shaped for people and it must be began with supporting users and grabbing advanced and fundamental needs of them.

The following figure shows the recent fears of technology and the predicted risk towards each life sector:

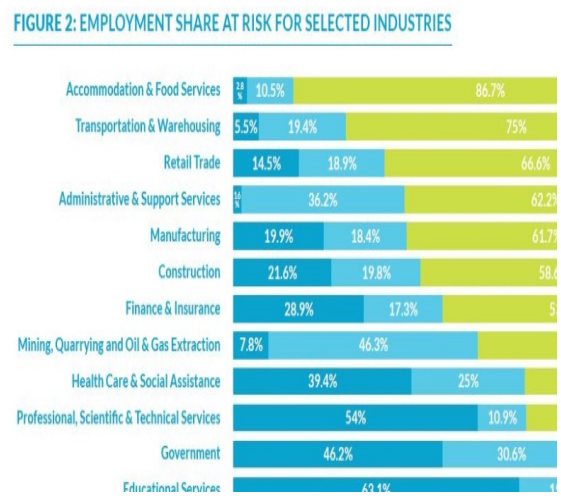

Fig6. Employment share at risks. 
From the figure we notice that, green area indicates the high risk percentage. Accommodation and food services sector has the greatest percentage of risk.

\section{Conclusion:}

This section summarizes the main points of this research study. The major aim of the study is to emphasize the essential tasks of software engineering and the main role of software engineers to improve this field in the future. The paper introduced main four areas, Internet of things, artificial intelligence, ubiquitous computing, and system of systems. We presented the recent view and challenges for each area and the future view and challenges as well.

Following the present directions and ways of using technology in the daily lives, we guess that the future would follow the same directions through using software in all fields and actions. In addition to surveying the state of art, we designed an online interview for experts from the same field. Six questions were assigned to the participants, 72 responds were received and analyzed. The majority of the answers reflected both of positive and negative attitudes towards the growth of technology through the four areas.

It can be concluded that, by 2050 many changes would be done on our daily and career lives because of the great development in software systems. Productivity will get higher and more qualified; the cost of software would be less, a number of jobs would disappear, and the privacy would be lost.

As a software engineer, I suggest having a huge system which can manage the produced amount of data every day. This system manages some sub-systems and each of them has its own manager to organize data and give accurate results for all tasks.

\section{References}

[1]F. Stallinger, "Software process simulation to support ISO/IEC 15504 based software process improvement", Software Process: Improvement and Practice, vol. 5, no. 23, p. 197, 2000.

[2]B. Fitzgerald and K. Stol, "Continuous software engineering: A roadmap and agenda", Journal of Systems and Software, vol. 123, pp. 176-189, 2017.

[31]H. W. B. Langdon and K. Y. Zhang, "Search based software engineering for software product line engineering: a survey and directions for future work", ACM SIGSOFT Software Engineering Notes, vol. 26, no. 3, pp. 118-125, 2015.

[4]A. Omicini, "Nature-Inspired Coordination Models: Current Status and Future Trends", ISRN Software Engineering, vol. 2013, pp. 1-13, 2013.

[5]Abdel-Hamid, T.K., Sengupta, K. and Ronan, D. Software project control: an experimental investigation of judgement with fallible information, IEEE Transactions on Software Engineering, 19(6):603-612, 1993

[6] Anda, B.C.D., Benestad, H.C. and Hove, S.E., A Multiple-Case Study of Effort Estimation based on Use Case Points, In ISESE'2005. IEEE Computer Society, Noosa, Australia, Nov. 17-18, pp. 407-416, 2005.

[7]"Appreciation to Empirical Software Engineering Reviewers of 2017", Empirical Software Engineering, 2018. [8]B. Fitzgerald and K. Stol, "Continuous software engineering: A roadmap and agenda", Journal of Systems and Software, vol. 123, pp. 176-189, 2017.

[9]F. Hermans, J. Siegmund, T. Fritz, G. Bavota, M. Nagappan, A. Hindle, Y. Kamei, A. Mesbah and B. Adams, "Leaders of Tomorrow on the Future of Software Engineering: A Roundtable", IEEE Software, vol. 33, no. 2, pp. 99-104, 2016.

[10]C. Chang, S. Srirama and R. Buyya, "Mobile Cloud Business Process Management System for the Internet of Things", ACM Computing Surveys, vol. 49, no. 4, pp. 1-42, 2016.

[11]G. Akpakwu, B. Silva, G. Hancke and A. Abu-Mahfouz, "A Survey on 5G Networks for the Internet of Things: Communication Technologies and Challenges", IEEE Access, vol. 6, pp. 3619-3647, 2018.

[12]S. Lee, M. Bae and H. Kim, "Future of IoT Networks: A Survey", Applied Sciences, vol. 7, no. 12, p. 1072, 2017.

[13]E. Daniel Rock, and Chad Syverson, "Artificial Intelligence and the Modern Productivity Paradox: A Clash of Expectations and Statistics", Business Economics, vol. 52, no. 2, pp. 99-102, 2017.

[14]G. Abowd and E. Mynatt, "Charting past, present, and future research in ubiquitous computing", ACM Transactions on Computer-Human Interaction, vol. 7, no. 1, pp. 29-58, 2000.

[15] Weiser, M. The computer for the 21st Century. Sci. Am. 265, 3 (Sept.1991), 94-104.

[16]A. Fano and A. Gershman, "The future of business services in the age of ubiquitous computing", Communications of the ACM, vol. 45, no. 12, 2002.

[17]P. Varga, F. Blomstedt, L. Ferreira, J. Eliasson, M. Johansson, J. Delsing and I. Martínez de Soria, "Making 
system of systems interoperable - The core components of the arrowhead framework", Journal of Network and Computer Applications, vol. 81, pp. 85-95, 2017.

[18]L. Martinez, J. Lambert and C. Karvetski, "Scenario-informed multiple criteria analysis for prioritizing investments in electricity capacity expansion", Reliability Engineering \& System Safety, vol. 96, no. 8, pp. 883$891,2011$.

[19]M. Zuckerberg, Privacy no longer a social norm, says Facebook founder. Eg. Youtube: technology, 2018.

[20]"A SURVEY: SMART PARKING SYSTEM USING INTERNET OF THINGS (IOT)", International Journal of Advance Engineering and Research Development, vol. 4, no. 04, 2017. 